\title{
BOUNDARY INTERPOLATION SETS FOR HOLOMORPHIC FUNCTIONS SMOOTH TO THE BOUNDARY AND BMO \\ BY \\ JOAQUIM BRUNA
}

\begin{abstract}
Let $\boldsymbol{A}^{p}$ denote the class of holomorphic functions on the unit disc whose first $p$-derivatives belong to the disc algebra. We characterize the boundary interpolation sets for $A^{p}$, that is, those closed sets $E \subset T$ such that every function in $C^{p}(E)$ extends to a function in $A^{p}$.

We also give a constructive proof of the corresponding result for $A^{\infty}$ (see [1]).

We show that the structure of these sets is in some sense related to BMO and that this fact can be used to obtain precise estimates of outer functions vanishing on $E$.
\end{abstract}

I. Introduction and summary of results. Let $D$ denote the open unit disc in the complex plane and $T$ its boundary. Let $A$ denote the algebra of functions analytic on $D$ and continuous on $\bar{D}$. For $p$ a positive integer, we denote by $A^{p}$ the algebra of functions $f$ such that $f$ and its first $p$ derivatives belong to $A$. If $s$ is positive and noninteger and $p=[s], A^{s}$ denotes the subspace of $A^{p}$ consisting of those $f \in A^{p}$ such that $f^{(p)}$ satisfies a Lipschitz condition of order $s-p$ :

$$
\left|f^{(p)}(z)-f^{(p)}(w)\right| \leqslant \text { const }|z-w|^{s-p} .
$$

Finally, we denote by $A^{\infty}$ the algebra of functions $f$ such that $f$ and all its derivatives belong to $A$.

If $f$ is in one of these algebras, its restriction to $T$ belongs, respectively, to the corresponding spaces $C(T), C^{p}(T), \operatorname{Lip}(s, T)$ or $C^{\infty}(T)$. A closed set $E \subset T$ is said to be a boundary interpolation set (B.I. sets, for short), for $A$ (resp. $A^{p}, A^{s}, A^{\infty}$ ) if given any $\phi \in C(T)$ (resp. $C^{p}(T), \operatorname{Lip}(s, T), C^{\infty}(T)$ ), there exists $f$ in $A$ (resp. $\left.A^{p}, A^{s}, A^{\infty}\right)$ such that

$$
\left(d^{j} f / d t^{j}\right)\left(e^{i t}\right)=\left(d^{j} \phi / d t^{j}\right)\left(e^{i t}\right)
$$

for all $e^{i t} \in E, j=0$ in the $A$-case, $j=0, \ldots, p$ in the $A^{p}$ and $A^{s}$-case, and all $j$ in the $A^{\infty}$-case.

The well-known Rudin-Carleson theorem states that the B.I.-sets for $A$ are exactly the sets of Lebesgue measure zero.

Definitions. Let $E \subset T$ be a closed set and $\rho(z)$ the Euclidean distance from $z$ to $E$. $E$ is said to be an $A T W$-set if it satisfies the condition

Received by the editors December 13, 1979.

AMS (MOS) subject classifications (1970). Primary 30A80; Secondary 42A40.

Key words and phrases. Boundary interpolation sets, Carleson sets, Lipschitz conditions, BMO, BMOA, outer functions. 
(ATW) "For all arcs $I \subset T$,

$$
\frac{1}{|I|} \int_{I} \log \frac{1}{\rho\left(e^{i t}\right)} d t \leqslant \text { const } \log \frac{1}{|I|}+\text { const” }
$$

where $|I|$ denotes the length of $I$. A closed set $E \subset T$ is said to be a $K$-set if it satisfies the condition

(K) "For all arcs $I \subset T$,

$$
\sup \{\rho(x), x \in I\} \geqslant \text { const }|I| ” .
$$

In [1], H. Alexander, B. A. Taylor and D. L. Williams proved that the B.I.-sets for $A^{\infty}$ are exactly the ATW-sets. In [5], E. M. Dynkin has shown that the B.I.-sets for $A^{s}$ are the $K$-sets.

The $A^{p}$-case is, as far as we know, an open problem. The proof of the Alexander-Taylor-Williams result uses duality arguments and the fact that the dual of $A^{\infty}$ can be represented as a space of analytic functions outside the disc. The proof of Dynkin's theorem uses an integral representation of functions in $\operatorname{Lip}(s, T)$ and the fact that this space is closed under conjugation (Privalov's theorem). There are no corresponding results in the $A^{p}$-case and that is why the proof of these theorems seems to have no extension to the integer case.

The main result of this paper is the characterization of B.I.-sets in the $A^{p}$-case. We show that they are also the $K$-sets and, in particular, do not depend on $p$, as it was to be expected.

Next, we briefly explain the contents of each section and some other relevant results.

In §II, together with the general notations we collect the auxiliary results used in the other sections.

The aim of $\S$ III is the construction, for a given $K$-set $E$ and for each $r>0$, of outer functions $F_{r}$ such that $\left|F_{r}(z)\right|$ is equivalent to $\rho(z)^{r}$ and $\left|F_{r}^{(k)}(z)\right|$ is dominated by $\rho(z)^{r-k}$. This construction is the main step in the proof of the main theorem. This result is already present in Dynkin's paper [5], using some function theory on Lipschitz domains. Our contribution in this section is to prove these estimates just using the classical function theory in the unit disc. We use that $\log \rho \in \mathrm{BMO}$ for $K$-sets and apply usual BMO techniques. Some other conditions equivalent to condition (K) are also found. ${ }^{1}$

We prove the main result in $\S I V$. We also note here that our method applies as well to the $A^{s}$-case and point out the changes to be done. The result has been announced in [12].

In $\S \mathrm{V}$ we give a new and constructive proof of the Alexander-Taylor-Williams theorem.

It is easy to see that every $K$-set is an ATW-set. In $\S$ VI we show, by exhibiting an example, that the converse is not true. The same example shows that $\log \rho \in \mathrm{BMO}$ is not a sufficient condition for $A^{p}$-interpolation.

\footnotetext{
${ }^{1}$ We must point out that the use of concepts related to BMO, for instance the Muckenhoupt weights, also appears in S. V. Hruscev, Sets of uniqueness for the Gevrey classes, Ark. Mat. 15 (1977), 253-304.
} 
II. Notations, definitions and auxiliary results. $E$ will always denote a closed set and $\rho(z)$ the Euclidean distance from $z$ to $E$. The letter $p$ will mean a positive integer and $s$ a positive noninteger real number, as used in the introduction.

A set $E$ is called a Carleson set if $\log \rho \in L^{1}(T)$. Carleson sets are exactly the boundary zero sets of functions in $A^{p}, A^{s}$ or $A^{\infty}$ (see [3], [10], [11]).

$A^{p}$ is a Banach space with the norm

$$
\|f\|_{A^{p}}=\sup \left\{\left|f^{(n)}(z)\right|, 0 \leqslant n \leqslant p, z \in \bar{D}\right\}
$$

and $A^{\infty}$ is a Fréchet space with the norms \|\|$_{A^{p}}, p>0$.

If $I$ is an arc in $T$, we denote by $|I|$ its length, and put

$$
R(I)=\{z \in D: z /|z| \in I \text { and } 1-|I| / 2 \pi<|z| \leqslant 1\} .
$$

The arc which has the same center as $I$ and $2^{n}$ times its length is denoted by $2^{n} I$.

We say that an $\operatorname{arc} I \subset T$ and a point $z \in D$ are related if $z /|z|$ is the center of $I$ and $|I|=1-|z|$.

For $u \in L^{1}(T)$, we denote by $I(u)$ the mean of $u$ over $I$,

$$
I(u)=\frac{1}{|I|} \int_{I} u d m, \quad d m=\text { Lebesgue measure on } T .
$$

BMO is the subspace of $L^{1}(T)$ of all $u \in L^{1}(T)$ such that

$$
\|u\|_{B M O}=\sup _{I \subset T} I(|u-I(u)|)<\infty
$$

that is, $u$ has bounded mean oscillation. The complex version of BMO is the space BMOA of all $f \in H^{1}$ whose boundary values belong to BMO.

If $x, y$ are two variables, the notation $x \sim y$ will mean that they are of the same order, in the sense that some constants $m, M>0$ exist such that $m<x / y<M$.

Finally, the notations "const" of $C, C_{n}$ mean positive constants, not necessarily the same at each occurrence.

We already know that $\log \left|e^{i t}-1\right|$ is in BMO. The following lemma gives a criterion for $\log \rho \in \mathrm{BMO}$, and will be used in the next section.

2.1. Lemma. Suppose $E$ is a Carleson set and that there exists $\alpha>0$ such that, for all $I \subset T$,

$$
m\{x \in I: \rho(x) \leqslant r\} \leqslant \text { const }|I|(r /|I|)^{\alpha}, \quad r>0 .
$$

Then, $\log \rho \in B M O$.

Proof. As in [6, p. 416], it is enough to find for each $I \subset T$ a value $a_{I}$ such that, if $S_{\sigma}=\left\{x \in I:\left|\log \rho(x)-a_{I}\right| \geqslant \sigma\right\}$,

$$
m\left(S_{\sigma}\right) \leqslant \text { const } e^{-b \sigma}|I|, \quad \text { for } \sigma>0,
$$

where $b$ does not depend on $I$. Given $I$, let $\xi, \eta \in I$ be such that

$$
\rho(\xi)=\sup \{\rho(x), x \in I\}, \quad \rho(\eta)=\inf \{\rho(x), x \in I\}
$$

and take $a_{I}=\log \rho(\xi)$. Then $S_{\sigma}=\left\{x \in I: \rho(x) \leqslant e^{-\sigma} \rho(\xi)\right\}$. If $S_{\sigma}$ is not empty, $\eta \in S_{\sigma}$ and $e^{-\sigma} \rho(\xi) \geqslant \rho(\eta) \geqslant \rho(\xi)-|\xi-\eta| \geqslant \rho(\xi)-|I|$. Thus

$$
\rho(\xi) \leqslant|I|\left(1-e^{-\sigma}\right)^{-1}, \quad S_{\sigma} \subset\left\{x \in I: \rho(x) \leqslant|I|\left(e^{\sigma}-1\right)^{-1}\right\}
$$


and using (1),

$$
m\left(S_{\sigma}\right) \leqslant \text { const }|I|\left(1 /\left(e^{\sigma}-1\right)\right)^{\alpha} .
$$

Since also $m\left(S_{\sigma}\right) \leqslant|I|$, we find (2).

It is well known that BMOA $\subset \mathscr{B}$, the Bloch space. That is, if $h \in \mathrm{BMOA}$, then $\left|h^{\prime}(z)\right|=O\left((1-|z|)^{-1}\right)$. More generally, we have the following result:

2.2. LEMMA. If $h \in B M O A$, then for $n \geqslant 1$,

$$
\left|h^{(n)}(z)\right|=O\left((1-|z|)^{-n}\right) .
$$

Proof. If $h \in \mathrm{BMOA}$, there exists $b \in L^{\infty}(T)$ whose analytic projection is $h$ (see [2, p. 22]) and

$$
\begin{aligned}
h^{(n)}(z) & =\frac{n !}{2 \pi i} \int_{|\xi|=1} \frac{b(\zeta)}{(\xi-z)^{n+1}} d \zeta=\frac{n !}{2 \pi} \int_{T} \frac{b\left(e^{i t}\right)}{\left(e^{i t}-z\right)^{n+1}} e^{i t} d t, \\
\left|h^{(n)}(z)\right| & \leqslant \text { const }\|b\|_{\infty} \int_{T} \frac{d t}{\left|e^{i t}-z\right|^{n+1}} .
\end{aligned}
$$

Let $I$ be related to $z$. An easy computation, estimating separately the contribution of $I$ and $T \backslash I$ in the last integral, shows that (3) holds.

Next lemma is, in fact, in [10]:

2.3. LemMA. Let $\psi$ be of class $C^{n+1}$ in some arc $J=[a, b]$ of $T$, let $c=(a+b) / 2$ the middle point of $J$ and let $A(z)$ be defined by

$$
A(z)=\int_{a}^{b} \frac{e^{i t}}{\left(e^{i t}-z\right)^{n+1}} \psi\left(e^{i t}\right) d t, \quad z \in D .
$$

Put $\psi^{0}=\psi$ and $\psi^{(k)}\left(e^{i t}\right)=e^{-i t}(d / d t) \psi^{(k-1)}\left(e^{i t}\right), k=1, \ldots, n+1$. Then, for $z=$ $r e^{i c}, 0 \leqslant r<1$,

$$
|A(z)| \leqslant \operatorname{const}\left(\sum_{k=0}^{n-1} \frac{M_{k}}{\left|z-e^{i a}\right|^{n-k}}+M_{n}+|J| M_{n+1}\right)
$$

where $M_{k}=\max \left\{\left|\psi^{(k)}\left(e^{i t}\right)\right|, a \leqslant t \leqslant b\right\}$.

Proof. Integrating by parts $n$ times we obtain

$$
\begin{aligned}
A(z)= & -\sum_{k=0}^{n-1} \frac{(n-k-1) !}{i^{k+1} \pi}\left[\frac{\psi^{(k)}\left(e^{i b}\right)}{\left(e^{i b}-z\right)^{n-k}}-\frac{\psi^{(k)}\left(e^{i a}\right)}{\left(e^{i a}-z\right)^{n-k}}\right] \\
& +\frac{1}{i^{n} \pi} \int_{b}^{a} \frac{e^{i t}}{\left(e^{i t}-z\right)} \psi^{(n)}\left(e^{i t}\right) d t .
\end{aligned}
$$

The first term in this expression gives the first one in (4). In the second one, write $\psi^{(n)}\left(e^{i t}\right)=\psi^{(n)}\left(e^{i c}\right)+\varepsilon(t)$. By the mean value theorem, $|\varepsilon(t)|<M_{n+1}|t-c|$. Thus, this term is less than

$$
\left|\psi^{(n)}\left(e^{i c}\right)\right|\left|\int_{a}^{b} \frac{e^{i t}}{e^{i t}-z} d t\right|+M_{n+1} \int_{a}^{b} \frac{|t-c|}{\left|e^{i t}-z\right|} d t \leqslant \text { const } M_{n}+M_{n+1}|J|
$$




\section{Properties of $K$-sets and construction of outer functions.}

3.1. Theorem. Let $E \subset T$ be a Carleson set and let $\rho(z)$ denote the distance between $z$ and $E$. The following statements are equivalent:

(a) For all $I \subset T, I(\log \rho) \geqslant \log |I|-$ const.

(b) $E$ is a $(K)$-set.

(c) There exists $0<\alpha<1$ such that $I\left(\rho^{-\alpha}\right) \leqslant$ const $|I|^{-\alpha}$, for all $I \subset T$.

(d) There exists $0<\alpha<1$ such that $I\left(\rho^{\alpha}\right) \geqslant$ const $|I|^{\alpha}$, for all $I \subset T$.

(e) There exists $0<\alpha<1$ such that, for all $I \subset T$,

$$
m\{x \in I: \rho(x) \leqslant r\} \leqslant \text { const }|I|(r /|I|)^{\alpha}, \quad r>0 .
$$

Proof. Write $s_{I}=\sup \{\rho(x), x \in I\}$. Remember that $E$ is a $K$-set if $|I| \leqslant$ const $s_{I}$.

(a) $\Rightarrow$ (b). This follows from $I(\log \rho) \leqslant \log s_{I}$.

(b) $\Rightarrow($ c). This is proved in [5, corollary to Lemma 2, p. 120].

(c) $\Rightarrow$ (a). By Jensen's inequality,

$$
\alpha I(-\log \rho)=I\left(\log \rho^{-\alpha}\right) \leqslant \log I\left(\rho^{-\alpha}\right) \leqslant \text { const }-\alpha \log |I|,
$$

and dropping away the $\alpha$, we have (a).

(c) $\Rightarrow$ (d). Just use Hölder's inequality: $1 \leqslant I\left(\rho^{\alpha}\right) I\left(\rho^{-\alpha}\right)$.

(d) $\Rightarrow$ (b). This follows from $I\left(\rho^{\alpha}\right) \leqslant s_{I}^{\alpha}$.

(c) $\Rightarrow$ (e). Let $T_{r}$ be the set appearing in (5). Then,

$$
\text { const }|I|^{-\alpha} \geqslant \frac{1}{|I|} \int_{I} \rho^{-\alpha} \geqslant \frac{1}{|I|} \int_{T_{r}} \rho^{-\alpha} \geqslant r^{-\alpha} \frac{1}{|I|} m\left(T_{r}\right)
$$

and (5) follows.

(e) $\Rightarrow$ (b). Just put $r=s_{I}$ in (5).

Condition (a) gives in particular that every $K$-set is an ATW-set. Examples of $K$-sets and their relation with ATW-sets will be given in the last section. From condition (c) one can obtain the following corollary, whose proof is in [5]:

3.2. Corollary. If $E$ is a $K$-set, for every arc $I \subset T$,

$$
\iint_{R(I)} \rho(\zeta)^{-1-\alpha} d \xi d \eta \leqslant \text { const }|I|^{1-\alpha}, \quad \zeta=\xi+i \eta
$$

where $\alpha$ is the same as in (c) of Theorem 3.1.

3.3. Corollary. If $E$ is a $K$-set and $\alpha$ is the same as in (c) of Theorem 3.1,

$$
\iint_{D} \frac{\rho(\zeta)^{-1-\alpha}}{|\zeta-z|} d \xi d \eta \leqslant \text { const } \rho(z)^{-\alpha}, \quad z \in D .
$$

Proof. First of all, observe that the left member is defined for all $z \in D$ and is a continuous function of $z$. This is because $|\zeta-z|^{-1}$ is locally integrable and $\rho(\zeta)^{-1-\alpha}$ is bounded near $z$ and, also, because near $E$, where $|\zeta-z|^{-1}$ is bounded, $\rho(\zeta)^{-1-\alpha}$ is integrable, by Corollary 3.2.

Break the integral into two parts $A$ and $B$, defined respectively by $|\zeta-z|<$ $\rho(z) / 2$ and $|\zeta-z| \geqslant \rho(z) / 2$. Now, $|\zeta-z| \leqslant \rho(z) / 2$ implies $\rho(\zeta) \geqslant \rho(z) / 2$. 
Hence,

$$
A \leqslant \text { const } \rho(z)^{-1-\alpha} \iint_{|\zeta-z|<\rho(z) / 2} \frac{1}{|\zeta-z|} d \xi d \eta<\text { const } \rho(z)^{-\alpha} .
$$

To estimate $B$, write $U_{k}=\left\{\zeta: 2^{k} \leqslant 2|\zeta-z| / \rho(z) \leqslant 2^{k+1}\right\}$. Then,

$$
B \leqslant \sum_{k=0}^{\infty} \iint_{U_{k}} \frac{\rho(\zeta)^{-1-\alpha}}{|\zeta-z|} d \xi d \eta \leqslant \sum_{k=0}^{\infty} \frac{1}{\rho(z) 2^{k-1}} \iint_{U_{k}} \rho(\zeta)^{-1-\alpha} d \xi d \eta
$$

The projection of the disc $|\zeta-z|<2^{k} \rho(z)$ on $T$ is an arc of length $<$ $2^{k} \rho(z)|z|^{-1}$. Also, the point of this disc nearest to 0 has distance $1-|z|+2^{k} \rho(z)<$ $\left(2^{k}+1\right) \rho(z)$ from $T$. Since it is enough to obtain the estimate for $|z|>1 / 2$, we see that this disc, and also $U_{k}$, is contained in a $R\left(I_{k}\right)$, with $\left|I_{k}\right| \leqslant 2^{k+1} \rho(z)$. Therefore, applying Corollary 3.2 , we get

$$
B \leqslant \text { const } \sum_{k=0}^{\infty} \frac{1}{\rho(z) 2^{k-1}}\left(2^{k+1} \rho(z)\right)^{1-\alpha}=\text { const } \rho(z)^{-\alpha} .
$$

From condition (e) in Theorem 3.1 and Lemma 2.1 we get:

\subsection{Corollary. Let $E$ be a $K$-set. Then $\log \rho \in B M O$.}

In the next theorem we shall need a modification of $\rho$ on $T$, which is equivalent to $\rho$ and is $C^{\infty}$ outside $E$. This can be obtained as follows (see also [11]). If $\left(a_{n}, b_{n}\right)$ are the complementary intervals of $E$ in $T$, define $\tilde{\rho}$ in $\left(a_{n}, b_{n}\right)$ by

$$
\tilde{\rho}(t)=\left(t-a_{n}\right)\left(b_{n}-t\right) /\left(b_{n}-a_{n}\right) .
$$

Then $\tilde{\rho} \sim \rho$. This implies $|I(\log \tilde{\rho})-I(\log \rho)| \leqslant$ const for every $I$, and therefore, $\log \tilde{\rho} \in$ BMO.

3.5. TheOREM. Let E be a $K$-set and $\tilde{\rho}$ defined as above. Let $h(z)$ be defined by

$$
h(z)=\frac{1}{2 \pi} \int_{0}^{2 \pi} \frac{e^{i t}+z}{e^{i t}-z} \log \tilde{\rho}\left(e^{i t}\right) d t, \quad z \in D,
$$

and let $u(z)$ be the real part of $h(z)$ (the Poisson transform of $\log \tilde{\rho})$. Define $F(z)=\exp h(z)$. Then, the following estimates hold:

(a) $|u(z)-\log \rho(z)| \leqslant$ const, $|F(z)| \sim \rho(z), z \in D$.

(b) For $n \geqslant 1,\left|F^{(n)}(z)\right| \leqslant C_{n} \rho(z)^{1-n}, z \in D$.

Proof. To prove (a), we use the fact that for a harmonic function $u$ with boundary values in BMO and for $I$ related to $z$, one has $|u(z)-I(u)|<$ const $\|u\|_{\text {BMO }}[2$, p. 6]. So it is enough to prove that $|\log \rho(z)-I(\log \tilde{\rho})|<$ const if $I$ and $z$ are related. Since $|I(\log \tilde{\rho})-I(\log \rho)| \leqslant$ const, we must see that

$$
|\log \rho(z)-I(\log \rho)| \leqslant \text { const }
$$

for $I$ related to $z$. There exists a constant $c$ such that $\left|e^{i t}-z\right| \leqslant c(1-|z|)$ for $e^{i t} \in I$. Hence $\rho\left(e^{i t}\right) \leqslant \rho(z)+\left|e^{i t}-z\right| \leqslant(c+1) \rho(z)$ on $I$ and so $I(\log \rho)<$ $\log \rho(z)+$ const. To obtain the reverse inequality, we distinguish two cases. If $c(1-|z|) \leqslant \rho(z) / 2$, then $\rho\left(e^{i t}\right) \geqslant \rho(z) / 2$ on $I$ and we get $I(\log \rho) \geqslant \log \rho(z)-$ const. If $\rho(z) / 2 \leqslant c(1-|z|)=c|I|$, we use (a) of Theorem 3.1 to obtain again

$$
I(\log \rho) \geqslant \log \rho(z)-\text { const. }
$$


This relation and the one above give (6) and (a). Since $|F(z)|=\exp u(z)$, it follows that $|F(z)| \sim \rho(z)$.

We will prove now that

$$
\left|h^{(n)}(z)\right| \leqslant C_{n} \rho(z)^{-n}, \quad z \in D .
$$

Then (b) will follow inductively from the relation $F^{\prime}=h^{\prime} F$ and (a). Since $\log \rho \in$ $\mathrm{BMO}$ and $\mathrm{BMO}$ is closed under conjugation, $h$ is in BMOA.

Fix some $c>1$. If $\rho(z) / 2 \leqslant c(1-|z|)$, (7) follows from Lemma 2.2.

If $\rho(z) / 2 \geqslant c(1-|z|)$, consider the arc $J=\left\{e^{i t}:\left|e^{i t}-z\right|<\rho(z) / 2\right\}$ of $T$. Let $a$, $b$ be the endpoints of $J$, so that $|a-z|=|b-z|=\rho(z) / 2$. We claim the following:

1. $|J| \sim \rho(z)$.

2. $\rho\left(e^{i t}\right) \sim \rho(z)$ for $e^{i t} \in J$.

3. $\left|e^{i t}-z\right| \sim\left|e^{i t}-c_{J}\right|$ for $e^{i t} \in J$, where $c_{J}$ is the center of $J$.

To prove $1,|J| \leqslant$ const $|a-b| \leqslant \operatorname{const}(|a-z|+|z-b|)<$ const $\rho(z)$. On the other side,

$$
\rho(z) / 2=|a-z| \leqslant\left|a-c_{J}\right|+1-|z| \leqslant\left|a-c_{J}\right|+\rho(z) / 2 c
$$

and so $\left|a-c_{J}\right| \geqslant \rho(z)(c-1) / 2 c$, which gives $|J| \geqslant$ const $\rho(z)$.

Point 2 is trivial. For the point 3 , if $\left|e^{i t}-z\right| \geqslant \rho(z) / 2$, one has

$$
\begin{aligned}
\left|e^{i t}-z\right| & \leqslant\left|e^{i t}-c_{J}\right|+1-|z| \leqslant\left|e^{i t}-c_{J}\right|+\rho(z) / 2 c \\
& \leqslant\left|e^{i t}-c_{J}\right|+c^{-1}\left|e^{i t}-z\right| .
\end{aligned}
$$

Hence,

$$
\left|e^{i t}-c_{J}\right| \geqslant((c-1) / c)\left|e^{i t}-z\right| .
$$

Also, $\left|e^{i t}-c_{J}\right| \leqslant 2\left|e^{i t}-z\right|$.

Now we write

$$
\begin{aligned}
h^{(n)}(z) & =\frac{n !}{\pi} \int_{0}^{2 \pi} \frac{e^{i t}}{\left(e^{i t}-z\right)^{n+1}} \log \tilde{\rho}\left(e^{i t}\right) d t \\
& =\frac{n !}{\pi} \int_{0}^{2 \pi} \frac{e^{i t}}{\left(e^{i t}-z\right)^{n+1}}\left(\log \tilde{\rho}\left(e^{i t}\right)-J(\log \tilde{\rho})\right) d t .
\end{aligned}
$$

Break this integral into two parts corresponding to $J$ and $T \backslash J$. To evaluate the contribution of $J$, we use Lemma 2.3 with $\psi\left(e^{i t}\right)=\log \tilde{\rho}\left(e^{i t}\right)-J(\log \tilde{\rho})$. With the notations of Lemma 2.3, point 2 above implies that $M_{0}<$ const. A direct computation shows that $M_{k} \sim \rho(z)^{-k}$ for $k \geqslant 1$. Thus, in any case, $M_{k}<C_{k} \rho(z)^{-k}$. Hence, by (4),

$$
\begin{aligned}
& \left|\int_{J} \frac{e^{i t}}{\left(e^{i t}-z\right)^{n+1}}\left(\log \tilde{\rho}\left(e^{i t}\right)-J(\log \tilde{\rho})\right) d t\right| \\
& \leqslant C_{n} \sum_{k=0}^{n-1} \frac{\rho(z)^{-k}}{\rho(z)^{n-k}}+\rho(z)^{-n}+\rho(z)^{-n} \leqslant C_{n} \rho(z)^{-n} .
\end{aligned}
$$

Here we have used that $|z-a|=\rho(z) / 2$ and point 1 . 
Now we evaluate the contribution of $T \backslash J$. By point 3,

$$
\begin{array}{r}
\left|\frac{n !}{\pi} \int_{T \backslash J} \frac{e^{i t}}{\left(e^{i t}-z\right)^{n+1}}\left(\log \tilde{\rho}\left(e^{i t}\right)-J(\log \tilde{\rho})\right) d t\right| \\
\leqslant C_{n} \int_{T \backslash J} \frac{\left|\log \tilde{\rho}\left(e^{i t}\right)-J(\log \tilde{\rho})\right|}{\left|e^{i t}-c_{J}\right|^{n+1}} d t .
\end{array}
$$

Let $J_{k}=2^{k} J$ for $k=0, \ldots, N-1$, where $N$ is the smallest integer such that $2^{N}|I| \geqslant 2 \pi$. Let $J_{N}=T$. We have, since $\left|e^{i t}-c_{J}\right| \geqslant$ const $2^{k}|J|$ outside $J_{k-1}$, that the last integral is less than

$$
\begin{array}{r}
C_{n} \sum_{k=1}^{N} 2^{-k(n+1)}|J|^{-n-1} \int_{J_{k} \backslash J_{k-1}}\left|\log \tilde{\rho}\left(e^{i t}\right)-J(\log \tilde{\rho})\right| d t \\
\leqslant C_{n}|J|^{-n} \sum_{k=1}^{N} 2^{-k n} J_{k}(|\log \tilde{\rho}-J(\log \tilde{\rho})|) .
\end{array}
$$

But $\log \tilde{\rho}$ is in BMO, and so, $J_{k}(|\log \tilde{\rho}-J(\log \tilde{\rho})|) \leqslant(2 k+1)\|\log \tilde{\rho}\|_{\text {BMO }}($ see $[8, \mathrm{p}$. 79]). Therefore, the above expression is bounded by some constant times $|J|^{-n}$, and so, using point 1 , some constant times $\rho(z)^{-n}$. This shows that also the contribution of $T \backslash J$ satisfies an estimation like (7), and finishes the proof of the theorem.

Remark. If $E$ is a Carleson set and $h, F$ are defined as in Theorem 3.5, the derivatives of $h$ (and $F$ ) satisfy estimates of the type $\left|h^{(n)}(z)\right| \leqslant C_{n} \rho(z)^{-q_{n}}$ for some integer $q_{n}$. This can be easily shown using the same method (see [3] or [10]). The important point in Theorem 3.5 is that we can choose $q_{n}=n-1$ and also that $|F(z)|$ is not only dominated by $\rho(z)$, but equivalent to it.

In particular, we see that if $E$ is a $K$-set, then $P[\log \rho](z)$, the Poisson transform of $\log \rho$, is like $\log \rho$. The converse is also true, so that this property can be added to the list of equivalent conditions to $(\mathrm{K})$. Even more than this is true, as shown by the following result:

\subsection{TheOREM. Let E be a Carleson set such that}

$$
P[\log \rho](z) \geqslant \log (1-|z|)-\text { const. }
$$

Then, $E$ is a $K$-set.

Proof. We will see that (a) of Theorem 3.1 is verified, for $I$ small. Given $I$, take $z$ such that $z /|z|$ is the center of $I$ and $z$ is at distance $\pi|I|$ from the endpoints of $I$. A computation shows that $P_{z}\left(e^{i t}\right) \geqslant|I|^{-1}$ in $I$ and that $(1-|z|) \sim|I|$. Now, $c-$ $\log \rho$ is positive for some $c>0$. Hence,

$$
\begin{aligned}
c+I(-\log \rho) & =I(c-\log \rho) \leqslant \int_{I} P_{z}\left(e^{i t}\right)\left(c-\log \rho\left(e^{i t}\right)\right) d t \\
& \leqslant \int_{T} P_{z}\left(e^{i t}\right)\left(c-\log \rho\left(e^{i t}\right)\right) d t=c-P[\log \rho](z) \\
& \leqslant c-\log |I|+\text { const. }
\end{aligned}
$$

Now we state the theorem that will be used in the proof of the main result. In it, $F_{r}$ is just $F$ raised to the $r$ th power, i.e., $F_{r}(z)=\exp r h(z)$. Part (c) of this theorem 
follows from the fact that $\tilde{\rho}$ and $\log \tilde{\rho}$ are $C^{\infty}$ in $T \backslash E$ (the conjugate of a function which is of class $C^{\infty}$ in an arc is also of class $C^{\infty}$ in this arc).

3.7. TheOREM. Let $E$ be $a K$-set and let $\rho(z)$ denote the distance from $z$ to $E$. Then, for each $r>0$, there exists an outer function $F_{r}$ such that:

(a) $\left|F_{r}(z)\right| \sim \rho(z)^{r}, z \in \bar{D}$.

(b) $\left|F_{r}^{(n)}(z)\right| \leqslant C_{n} \rho(z)^{r-n}, z \in D, n \geqslant 1$.

(c) $F_{r} \in C^{\infty}(T \backslash E)$.

We note that the estimates in (b) also hold for $z \in T \backslash E$.

IV. Proof of the main result. In this section we will prove the following theorem:

4.1. TheOrem. If $p$ is a positive integer, a closed set $E \subset T$ is a boundary interpolation set for $A^{p}$ if and only if it is a $K$-set.

The necessity. Necessity is proved in the same way as in the $A^{s}$-case. We briefly explain, for the sake of completeness, how this proof goes. If $E$ is a (B.I.) set for $A^{p}$, for each $\zeta \in D$, the function $\phi_{\zeta}(z)=(\zeta-z)^{-1}$ must be interpolated by some $f_{\zeta} \in A^{p}$. But, using the open mapping theorem, we can obtain some control in the bounds

$$
\left\|f_{\zeta}\right\|_{A^{p}} \leqslant \text { const }\left\|\phi_{\zeta}\right\|_{C^{p}}=\operatorname{const}(1-|\zeta|)^{-(p+1)} .
$$

Write $g_{\zeta}(z)=1-(\zeta-z) f_{\zeta}(z)$, so that $g_{\zeta}(\zeta)=1$ and $g_{\zeta}$, as well as its first $p$ derivatives, vanishes on $E$. This latter fact implies

$$
\left|g_{\zeta}(z)\right| \leqslant\left\|g_{\zeta}\right\|_{A^{p}} \rho(z)^{p} \leqslant \operatorname{const}(1-|\zeta|)^{-(p+1)} \rho(z)^{p} .
$$

But this estimate can be improved to $\left|g_{\zeta}(z)\right| \leqslant \operatorname{const}(1-|\zeta|)^{-p} \rho(z)^{p}$ for $|\zeta-z| \leqslant$ const $(1-|\zeta|)$. In particular, this last estimate holds for $z$ in the curvilinear square centered at $\zeta$. Then, an application of Jensen's inequality leads to condition (K).

The sufficiency. For simplicity, we will prove the sufficiency of condition (K) just in case $p=1$, and comment on the general case at the end.

Let $\phi \in C^{1}(T)$. Writing $\phi_{1}\left(e^{i t}\right)=-i e^{-i t}(d \phi / d t)\left(e^{i t}\right), \phi_{2}\left(e^{i t}\right)=i \phi_{1}\left(e^{i t}\right), e^{i t} \in E$, it is easy to see that $\left(\phi, \phi_{1}, \phi_{2}\right)$ is a Whitney jet on $E$. Whitney's extension theorem allows us to suppose that $\phi \in C^{1}(\mathbf{C}), \phi \in C^{\infty}(\mathbf{C} \backslash E)$ and, by construction, that the continuous function $\Phi=\bar{\partial} \phi$ vanishes on $E$. The following estimates also hold, for $|\zeta| \leqslant 2, a \in E$ :

$$
\begin{array}{cl}
\left|D^{k} \phi(\zeta)-D^{k} \phi(a)\right| \leqslant \text { const } \omega(|\zeta-a|) & \text { for every bi-index } k,|k|=1 . \\
\left|D^{k} \phi(\zeta)\right| \leqslant \frac{C_{k} \omega(\rho(\zeta))}{\rho(\zeta)^{|k|-1}}, & |k|>1, \zeta \notin E .
\end{array}
$$

Here, $\omega$ is a modulus of continuity, that is, a nondecreasing function on $[0, \infty)$, concave and continuous, with $\omega(0)=0$. (See [7].) In particular,

$$
\begin{aligned}
|\Phi(\zeta)| \leqslant \text { const } \omega(\rho(\zeta)), & |\zeta| \leqslant 2, \\
|\partial \Phi(\zeta)| \leqslant \text { const } \frac{\omega(\rho(\zeta))}{\rho(\zeta)}, & |\zeta| \leqslant 2, \zeta \notin E .
\end{aligned}
$$


Consider now the function $F_{r}$ of Theorem 3.7, with $r=1+\alpha$, where $\alpha$ is the one appearing in Theorem 3.1 and corollaries. Solve now the equation $\bar{\partial} v=\Phi / F_{r}$. As we will see, one solution is

$$
v(z)=\frac{1}{2 \pi i} \iint_{D} \frac{\Phi(\zeta)}{F_{r}(\zeta)} \frac{d \xi d \eta}{\zeta-z}, \quad \zeta=\xi+i \eta, z \in D
$$

The result will be proved in three steps.

1. $v(z)$ is a continuous function on $\mathrm{C} \backslash E$ such that

$$
|v(z)|=o\left(\rho(z)^{-\alpha}\right) \quad \text { as } \rho(z) \rightarrow 0 .
$$

Fix $z \notin E$. Write $H(z)=\{\zeta:|\zeta| \leqslant 1,|\zeta-z| \leqslant \rho(z) / 2\}$. In the disc $|w-z|<$ $\rho(z) / 4$, we can write $v(w)$ in two parts:

$$
\begin{aligned}
& v(w)=\frac{1}{2 \pi i} \iint_{D \backslash H(z)} \frac{\Phi(\zeta)}{F_{r}(\zeta)} \frac{d \xi d \eta}{\zeta-w}+\frac{1}{2 \pi i} \iint_{H(z)} \frac{\Phi(\zeta)}{F_{r}(\zeta)} \frac{d \xi d \eta}{\zeta-w} \\
& \quad \stackrel{\text { def }}{=} A(w)+B(w) .
\end{aligned}
$$

In the first integral, $|\zeta-w| \geqslant \rho(z) / 4$. Also, $\Phi$ is bounded and $\left|F_{r}(\zeta)\right| \sim \rho(\zeta)^{r}$. Therefore, $A(w)$, for $|z-w|<\rho(z) / 4$, is dominated by

$$
\text { const } \rho(z)^{-1} \iint_{D} \rho(\zeta)^{-r} d \xi d \eta
$$

which is convergent, by Corollary 3.2. In particular, $A(w)$ is a continuous function on $|z-w|<\rho(z) / 4$.

In the second integral, $\rho(\zeta) \geqslant \rho(z) / 2$. Since $\left|F_{r}(\zeta)\right| \sim \rho(\zeta)^{r}$ and $\Phi$ is bounded we see that the integrand in $B(w)$ is bounded, and so, $B(w)$ is also continuous (Cauchy transform of a bounded function).

This proves that $v(z)$ is a continuous function on $\mathbf{C} \backslash E$. To prove (10), since $\left|F_{r}(\zeta)\right| \sim \rho(\zeta)^{r}$, it is enough to show that

$$
\iint_{D} \frac{|\Phi(\zeta)|}{\rho(\zeta)^{r}} \frac{d \xi d \eta}{|\zeta-z|} \leqslant \beta(\rho(z)) \rho(z)^{-\alpha}
$$

for some continuous function $\beta$, with $\beta(0)=0$. Break this last integral into two parts, corresponding to $\rho(\zeta) \geqslant \rho(z)^{\alpha / 2 r}$ and $\rho(\zeta) \leqslant \rho(z)^{\alpha / 2 r}$. The contribution of the first is less than

$$
\left(\rho(z)^{-\alpha / 2 r}\right)^{r} \iint_{D} \frac{|\Phi(\zeta)|}{|\zeta-z|} d \xi d \eta \leqslant \text { const } \rho(z)^{-\alpha / 2}
$$

and so satisfies a bound like (12). In the second part, where $\rho(\zeta)<\rho(z)^{\alpha / 2 r}$, one has $|\Phi(\zeta)| \leqslant$ const $\omega\left(\rho(z)^{\alpha / 2 r}\right)$, by (8). Hence, its contribution is less than const $\omega\left(\rho(z)^{\alpha / 2 r}\right) \iint_{D}\left(\rho(\zeta)^{-r} /|\zeta-z|\right) d \xi d \eta$. Now, Corollary 3.3 shows that this also satisfies a bound like (12). This ends the proof of the first step.

2. $v(z)$ is of class $C^{1}$ in $D, \bar{\partial} v=\Phi / F_{r}$, $\partial v$ has a continuous extension to $\bar{D} \backslash E$ and

$$
|\partial v(z)|=o\left(\rho(z)^{-1-\alpha}\right) \quad \text { as } \rho(z) \rightarrow 0, z \in \bar{D} \backslash E .
$$

Fix $z \in \bar{D} \backslash E$, and consider again the expression (11) of $v$ in $|w-z|<\rho(z) / 4$, $|w|<1$. Using Stokes' theorem, we can write the second term in (11) in another 
way. For $|w-z|<\rho(z) / 4,|w|<1$,

$$
\begin{aligned}
v(w)= & \frac{1}{2 \pi i}\left\{\iint_{D \backslash H(z)} \frac{\Phi(\zeta)}{F_{r}(\zeta)} \frac{d \xi d \eta}{\zeta-w}-\iint_{H(z)} \frac{\partial}{\partial \zeta} \frac{\Phi(\zeta)}{F_{r}(\zeta)} \log |\zeta-w|^{2} d \xi d \eta\right\} \\
& +\frac{1}{2 \pi i} \int_{\partial H_{(z)}} \frac{\Phi(\zeta)}{F_{r}(\zeta)} \log |\zeta-w|^{2} d \bar{\zeta}
\end{aligned}
$$

In this expression we can apply the operator $\bar{\partial}$ because the integrands that appear are still integrable. We get, since the first term is holomorphic,

$$
\bar{\partial} v(w)=\frac{1}{2 \pi i} \iint_{H(z)} \frac{\partial}{\partial \zeta} \frac{\Phi(\zeta)}{F_{r}(\zeta)} \frac{d \xi d \eta}{\bar{\zeta}-\bar{w}}-\frac{1}{2 \pi i} \int_{\partial H(z)} \frac{\Phi(\zeta)}{F_{r}(\zeta)} \frac{d \bar{\zeta}}{\bar{\zeta}-\bar{w}}
$$

By Cauchy's generalized formula, this result equals $\Phi / F_{r}$. Therefore, $\bar{\partial} v=\Phi / F_{r}$.

Now we apply $\partial$ on (14). We get

$$
\begin{aligned}
& \partial v(w)= \frac{1}{2 \pi i}\left\{\iint_{D \backslash H(z)} \frac{\Phi(\zeta)}{F_{r}(\zeta)} \frac{d \xi d \eta}{(\zeta-w)^{2}}\right. \\
&\left.+\iint_{H(z)} \frac{\partial \Phi(\zeta) F_{r}(\zeta)-F_{r}^{\prime}(\zeta) \Phi(\zeta)}{F_{r}^{2}(\zeta)} \frac{d \xi d \eta}{\zeta-w}\right\} \\
&-\frac{1}{2 \pi i}\left\{\int_{\gamma_{1}} \frac{\Phi(\zeta)}{F_{r}(\zeta)} \frac{d \zeta}{\zeta-w}+\int_{\gamma_{2}} \frac{\Phi(\zeta)}{F_{r}(\zeta)} \frac{d \zeta}{\zeta-w}\right\} \\
& \stackrel{\operatorname{def}}{=} C(w)+D(w)+E_{1}(w)+E_{2}(w) .
\end{aligned}
$$

Here, $\gamma_{1}=\{\zeta \in \bar{D}:|\zeta-z|=\rho(z) / 2\}$ and $\gamma_{2}=\{\zeta \in T,|\zeta-z| \leqslant \rho(z) / 2\}$ are the two possible parts of $\partial H(z)$. The expression (15) is valid for $|w|<1,|w-z|<$ $\rho(z) / 4$.

If $z \in T \backslash E$, it is clear that $C(w)$ and $D(w)$ have a continuous extension to the arc $\{w \in T:|w-z|<\rho(z) / 4\}$. This would be proved in the same way that we have proved that $A(w), B(w)$ were continuous functions in point 1 . That $E_{1}(w)$ also has a continuous extension to this arc is trivial. The function $E_{2}(w)$ also extends continuously to this arc. This is because $\Phi$ and $F_{r}$ are of class $C^{\infty}$ in $T \backslash E$ and the regularity on an arc of the Cauchy integral is the same as that of the conjugate function. Therefore, $\partial v(w)$ has a continuous extension to a neighbourhood of $z$. Since $z$ was arbitrary in $T \backslash E$, this proves that $\partial v$ extends continuously to $\bar{D} \backslash E$.

Now we turn to the proof of (13). It is enough to prove an estimate of the type

$$
|\partial v(z)| \leqslant \gamma(\rho(z)) \rho(z)^{-1-\alpha}, \quad z \in D
$$

for some continuous function $\gamma$, with $\gamma(0)=0$. To do this, we put $w=z$ in (15) and estimate separately $C(z), D(z), E_{1}(z)$ and $E_{2}(z)$.

We have

$$
\begin{aligned}
|C(z)| & \leqslant \text { const } \iint_{D \backslash H(z)} \frac{|\Phi(\zeta)|}{\rho(\zeta)^{r}} \frac{d \xi d \eta}{|\zeta-z|^{2}} \\
& \leqslant \text { const } \rho(z)^{-1} \iint_{D \backslash H(z)} \frac{|\Phi(\zeta)|}{\rho(\zeta)^{r}} \frac{d \zeta d \eta}{|\zeta-z|} .
\end{aligned}
$$

Now, (12) implies that $C(z)$ satisfies an estimate like (16). 
To estimate $D(z)$ and $E_{1}(z)$, we use (8), (9) and parts (a), (b) of Theorem 3.7. We get

$$
\begin{aligned}
& |D(z)| \leqslant \text { const } \iint_{H(z)} \frac{\omega(\rho(\zeta))}{\rho(\zeta)^{r+1}} \frac{d \xi d \eta}{|\zeta-z|} \\
& \left|E_{1}(z)\right| \leqslant \text { const } \int_{\gamma_{1}} \frac{\omega(\rho(\zeta))}{\rho(\zeta)^{r}} \frac{|d \bar{\zeta}|}{|\zeta-z|} .
\end{aligned}
$$

But $\rho(\zeta) \sim \rho(z)$ for $\zeta$ in $H(z)$. Hence,

$$
\begin{aligned}
& |D(z)| \leqslant \text { const } \omega(\rho(z)) \rho(z)^{-r-1} \iint_{H(z)}|\zeta-z|^{-1} d \xi d \eta \leqslant \text { const } \omega(\rho(z)) \rho(z)^{-r}, \\
& \left|E_{1}(z)\right| \leqslant \text { const } \omega(\rho(z)) \rho(z)^{-r} \int_{\gamma_{1}}|\zeta-z|^{-1}|d \bar{\zeta}| \leqslant \text { const } \omega(\rho(z)) \rho(z)^{-r}
\end{aligned}
$$

which are of type (16).

It remains to prove the estimate for $E_{2}(z)$. We can write

$$
E_{2}(z)=\frac{1}{2 \pi i} \int_{\gamma_{2}} \frac{\Phi(\zeta)}{\zeta^{2} F_{r}(\zeta)} \frac{d \zeta}{\zeta-z} .
$$

Now we use Lemma 2.3 with $\psi(\zeta)=\Phi(\zeta) / \zeta^{2} F_{r}(\zeta)$ (recall that $\Phi$ and $F_{r}$ are $C^{\infty}$ in $T \backslash E$ ) and $n=0$. With the notations of that lemma, using (8), (9), the properties of $F_{r}$ and the fact that $\rho(\zeta) \sim \rho(z)$ for $\zeta \in \gamma_{2}$, one gets that $M_{0} \leqslant$ const $\omega(\rho(z)) \rho(z)^{-r}$ and $M_{1} \leqslant$ const $\omega(\rho(z)) \rho(z)^{-1-r}$. Since the length of $\gamma_{2}$ is less than const $\rho(z)$ we finally obtain $\left|E_{2}(z)\right| \leqslant$ const $\omega(\rho(z)) \rho(z)^{-r}$.

Thus, proof of point 2 is finished.

3. Let us define now $f=\phi-v F_{r} . \bar{\partial} v=\Phi / F_{r}=\bar{\partial} \phi / F_{r}$ implies that $f$ is holomorphic in $D$. Taking into account the properties of $F_{r}$ and the points 1 and 2 , the functions $v(z) F_{r}(z), v(z) F_{r}^{\prime}(z)$ and $\partial v(z) F_{r}(z)$ extend continuously to $\bar{D} \backslash E$ and satisfy respectively

$$
\begin{aligned}
\left|v(z) F_{r}(z)\right| & =o(\rho(z)), \\
\left|v(z) F_{r}^{\prime}(z)\right| & =o(1), \\
\left|\partial v(z) F_{r}(z)\right| & =o(1),
\end{aligned}
$$

as $\rho(z) \rightarrow 0$. That is, these functions, extended by 0 on $E$, are continuous functions on $\bar{D}$. This amounts to saying that $f \in A^{1}$ and that $f$ interpolates $\phi$ and its derivative on $E$.

This proves the sufficiency of condition $(\mathrm{K})$ in case $p=1$.

REMARK. In case $p>1$, the assumptions (8), (9) on $\Phi$ would be replaced by

$$
\begin{aligned}
|\Phi(\zeta)| & \leqslant \text { const } \omega(\rho(\zeta)) \rho(\zeta)^{p-1}, \quad|\zeta| \leqslant 2, \\
\left|\partial^{(k)} \Phi(\zeta)\right| & \leqslant \text { const } \frac{\omega(\rho(\zeta))}{\rho(\zeta)^{k+1-p}}, \quad k \geqslant 1, \zeta \notin E .
\end{aligned}
$$

In this case we would take $r=p+\alpha$ and would prove that, for $1<k \leqslant p$, the function $\partial^{(k)} v(z)$ has a continuous extension to $\bar{D} \backslash E$ satisfying

$$
\left|\partial^{(k)} v(z)\right|=o\left(\rho(z)^{-\alpha-k}\right) \quad \text { as } \rho(z) \rightarrow 0 .
$$


This would be proved as in case $p=1$, using expression (15), together with the fact that $\Phi$ and $F_{r}$ are $C^{\infty}$ outside $E$. With (18) proved, we would replace the estimates (17) by $\left|\partial^{(k)} v(z) F_{r}^{(j)}(z)\right|=o\left(\rho(z)^{p-k-j}\right)$ and from these obtain that $f \in A^{p}$ and that $f$ interpolates $\phi$ and its first $p$ derivatives on $E$.

We show now that the above proof can be adapted to the $A^{s}$-case. We will do it in case $0<s<1$.

Given $\phi \in \operatorname{Lip}(s, T)$, we could suppose this time, using a theorem of Whitney's type (see [9]), that $\phi \in C^{\infty}(C \backslash E)$ and that if $\Phi=\bar{\partial} \phi$

$$
|\Phi(\zeta)| \leqslant \text { const } \rho(\zeta)^{s-1}, \quad|\partial \Phi(\zeta)| \leqslant \text { const } \rho(\zeta)^{s-2},
$$

for $|\zeta| \leqslant 2, \zeta \notin E$.

Take now $r=s+\alpha$ and define $v$ in the same way.

1. $v(z)$ is a continuous function on $\mathbf{C} \backslash E$ such that

$$
|v(z)|=O\left(\rho(z)^{-\alpha}\right) \quad \text { as } \rho(z) \rightarrow 0 .
$$

The continuity of $v$ is proved as before. The estimate (19) and (a) of Theorem 3.7 imply

$$
|v(z)| \leqslant \text { const } \iint_{D} \frac{\rho(\zeta)^{-1-\alpha}}{|\zeta-z|} d \xi d \eta
$$

and (20) follows from Corollary 3.3.

2. $v(z)$ is of class $C^{1}$ in $D, \bar{\partial} v=\Phi / F_{r}$ and

$$
|\partial v(z)|=O\left(\rho(z)^{-1-\alpha}\right) \quad \text { as } \rho(z) \rightarrow 0 .
$$

We just sketch how to obtain (21). We consider again expression (15) for $w=z$ and estimate separately $C(z), D(z), E_{1}(z)$ and $E_{2}(z)$.

For $C(z)$,

$$
|C(z)| \leqslant \text { const } \rho(z)^{-1} \iint \frac{\rho(\zeta)^{-1-\alpha}}{|\zeta-z|} d \xi d \eta \leqslant \text { const } \rho(z)^{-1-\alpha} .
$$

For $D(z)$ and $E_{1}(z)$, using (19) and Theorem 3.7,

$$
\begin{aligned}
& |D(z)| \leqslant \text { const } \iint_{H(z)} \frac{\rho(\zeta)^{-2-\alpha}}{|\zeta-z|} d \xi d \eta \\
& |E(z)| \leqslant \text { const } \int_{\gamma_{1}} \frac{\rho(\zeta)^{-1-\alpha}}{|\zeta-z|} d \bar{\zeta} .
\end{aligned}
$$

But $\rho(\zeta) \sim \rho(z)$ for $\zeta$ in $H(z)$. Then one gets

$$
\begin{aligned}
& |D(z)| \leqslant \text { const } \rho(z)^{-2-\alpha} \iint_{H(z)}|\zeta-z|^{-1} d \xi d \eta \leqslant \text { const } \rho(z)^{-1-\alpha}, \\
& |E(z)| \leqslant \text { const } \rho(z)^{-1-\alpha} \int_{\gamma_{1}}|\zeta-z|^{-1} d \bar{\zeta} \leqslant \text { const } \rho(z)^{-1-\alpha} .
\end{aligned}
$$

Finally, the estimate for $E_{2}(z)$ is also obtained using Lemma 2.3.

3. If $f=\phi-v F_{r}, f$ is in the disc algebra, because (20) implies that $v F_{r}$ extends continuously to $E$. Also, by (20), (21) and the properties of $F_{r},\left|\partial v(z) F_{r}(z)\right|=$ $O\left(\rho(z)^{s-1}\right),\left|v(z) F_{r}^{\prime}(z)\right|=O\left(\rho(z)^{s-1}\right)$. But also $\partial \phi(z)$ satisfies an estimate like $\Phi$ and 
so,

$$
\left|f^{\prime}(z)\right|=O\left(\rho(z)^{s-1}\right) .
$$

In particular, $\left|f^{\prime}(z)\right|=O\left((1-|z|)^{s-1}\right)$ and then the Hardy-Littlewood theorem [4] shows that the boundary values of $f$ are in $\operatorname{Lip}(s, T)$. But this is in fact equivalent to $f \in A^{s}$. Thus we can state (this is the theorem proved in [5]):

4.2. TheOREM. Let $s$ be a noninteger positive number. A set $E$ is a B.I.-set for $A^{s}$ if and only if it is a $K$-set.

V. The $A^{\infty}$-case. In this section we will give a constructive proof of the following theorem of H. Alexander, B. A. Taylor and D. L. Williams, quoted in the introduction:

5.1. Theorem. $A$ set $E$ is a B.I.-set for $A^{\infty}$ if and only if it is a $A T W$-set.

We will need the following lemma, which also gives the analogous result to Theorem 3.6 for the ATW-sets (see [1]):

5.2. Lemma. Let $u \in L^{1}(T)$, positive. Then the Poisson transform of $u$ satisfies

$$
P[u](z) \geqslant \text { const } \log (1-|z|)-\text { const }
$$

if and only if $I(u) \geqslant$ const $\log |I|-$ const, for every arc $I \subset T$.

Now we establish two other preliminary lemmas.

5.3. Lemma. Let $E$ be an $A T W$-set. Then for each $p \in N$ there exists an outer function $G_{p} \in A^{p}$ such that:

(a) $G_{p}^{(n)}(z)=0$ for $0 \leqslant n \leqslant p$ and $z \in E$.

(b) $\left|G_{p}(z)\right| \geqslant c_{p}(1-|z|)^{m}$ for some $m=m(p)$ and $c_{p}>0$.

(c) $G_{p} \in C^{\infty}(T \backslash E)$ and $\left|G_{p}^{(n)}(z)\right| \leqslant C_{n} \rho(z)^{-q_{n}}$ for some integer $q_{n}$.

Proof. We use the same notations as in Theorem 3.5. As said in the remark after that theorem, we have

$$
\left|h^{(n)}(z)\right| \leqslant C_{n} \rho(z)^{-q_{n}}
$$

for some $q_{n} \in N$. Define $G_{p}(z)=\exp Q_{p} h(z)$ where $Q_{p}$ is an integer. Part (c) follows from the fact that $\log \tilde{\rho} \in C^{\infty}(T \backslash E)$ and from (22). Also it is clear that if $Q_{p}$ is sufficiently large, we will have $\left|G_{p}^{(n)}(z)\right|=o(\rho(z))$ as $\rho(z) \rightarrow 0$ for $n \leqslant p$. Thus $G_{p} \in A^{p}$ and (a) holds. Finally, if $E$ is an ATW-set, the function $u=Q_{p} \log \tilde{\rho}$ satisfies the condition in Lemma 5.2, and (b) follows.

5.4. Lemma. Let $E$ be a Carleson set. Then for each $p \in N$ there exists $\bar{p} \in N$ with the following approximation property: if $g \in A^{\bar{p}}$ satisfies

(a) $g^{(n)}(z)=0$ for $n \leqslant \bar{p}$ and $z \in E$,

(b) $g \in C^{\infty}(T \backslash E)$ and $\left|g^{(n)}(z)\right| \leqslant C_{n} \rho(z)^{-q_{n}}$ for some integer $q_{n}$, and $\varepsilon>0$ is given, there exists $k \in A^{\infty}$ vanishing on $E$ together with all its derivatives and such that $\|g-k\|_{A^{p}} \leqslant \varepsilon$. Also, $\lim _{p \rightarrow \infty} \bar{p}=\infty$. 
Proof. In [11, pp. 127] it is proved there exists a sequence $\left(R_{k}\right)$ of functions in $A^{\infty}$ of the form $R_{k}=\exp -S_{k}$ such that

$$
\begin{gathered}
\qquad R_{k}^{(n)}(z)=0 \text { for all } n \text { and } z \in E . \\
\left|R_{k}(z)\right| \leqslant 1 \quad \text { and } \quad R_{k}(z) \rightarrow 1 \text { for every } z \in D \text {, as } k \rightarrow \infty . \\
\left|S_{k}^{(n)}(z)\right| \leqslant D_{n} \rho(z)^{-q_{n}} \text { for some constants } D_{n} \text { and } q_{n} .
\end{gathered}
$$

Take now $g \in A^{\bar{p}}$ satisfying (a) and (b). Since $g$ satisfies (b) and $R_{k}$ satisfies (23), we have $R_{k} g \in A^{\infty}$. So it is enough to prove that for $\bar{p}$ sufficiently large, $R_{k} g$ approaches $g$ in $A^{p}$ as $k$ goes to infinity. In view of (24), this would be true if $\left\{R_{k} g\right.$, $k \in N\}$ is a bounded subset of $A^{p+1}$. When computing $\left\|R_{k} g\right\|_{A^{p+1}}$, by (25), there appear expressions of the type $D_{n} \rho(z)^{-q_{n}}$ together with $\left|g^{(j)}(z)\right|, n, j$ from 0 to $p+1$. If $K_{p}=\max \left\{q_{n}, 0<n<p+1\right\}$, we will have $\sup _{k}\left\|R_{k} g\right\|_{A^{p+1}}<\infty$ as soon as $\left|g^{(j)}(z)\right|=O\left(\rho(z)^{K_{p}}\right)$ for $j=0, \ldots, p+1$. To insure this, in view of (a), it is enough to take $\bar{p}>p+1+K_{p}$.

Proof of Theorem 5.1. Necessity. Suppose that $E$ is a B.I.-set for $A^{\infty}$. By the open mapping theorem (for Fréchet spaces), for every $n \in N$ there exist an $m=m(n)$ and a constant $C_{n}>0$ such that, for every $\phi \in C^{\infty}(T)$, there exists $f \in A^{\infty}$ interpolating it and such that $\|f\|_{A^{n}} \leqslant C_{n}\|\phi\|_{C^{m}}$. Take $n=1$ and the function $\phi_{\zeta}(z)=(\zeta-z)^{-1}$ for $\phi$. Let $f_{\zeta}$ be the corresponding $f_{\zeta}$ and write $g_{\zeta}(z)=1$ - $(\zeta-z) f_{\zeta}(z)$. Then $\left\|g_{\zeta}\right\|_{A^{1}} \leqslant$ const $\left\|\phi_{\zeta}\right\|_{A^{m}}=\operatorname{const}(1-|\zeta|)^{-m-1}$ for some $m$. Also, $g_{\zeta}(\zeta)=1$ and $g_{\zeta}$ vanishes, together with all its derivatives, on $E$. In particular,

$$
\left|g_{\zeta}(z)\right| \leqslant \operatorname{const}(1-|\zeta|)^{-m-1} \rho(z)
$$

By Jensen's inequality,

$$
\begin{aligned}
\log \left|g_{\zeta}(z)\right| & \leqslant P\left[\log \left|g_{\zeta}\right|\right](z) \leqslant P[\text { const }-(m+1) \log (1-|\zeta|)+\log \rho](z) \\
& =\text { const }-(m+1) \log (1-|\zeta|)+P[\log \rho](z) .
\end{aligned}
$$

Putting $z=\zeta$ in this inequality one gets $P[\log \rho](z) \geqslant(m+1) \log (1-|\zeta|)-$ const and this is equivalent to condition (ATW), by Lemma 5.2.

Sufficiency. Suppose $E$ is an ATW-set and $\phi \in C^{\infty}(T)$.

1. For each $p \in N$, there exists $f_{p} \in A^{p}$ such that $f_{p}^{(n)}(z)=\phi^{(n)}(z)$ for $n \leqslant p$ and $z \in E$ (that is, $f_{p}$ interpolates the first $p$ derivatives of $\left.\phi\right)$. Also, $f_{p} \in C^{\infty}(T \backslash E)$ and $\left|f_{p}^{(n)}(z)\right| \leqslant C_{n} \rho(z)^{-q_{n}}$ for some integers $q_{n}$.

To prove point 1 , we take an almost analytic continuation of $\phi$. That is, the function $\Phi=\bar{\partial} \phi$ vanishes on $T$ together with all is derivatives (to do this, complete on $T$ the jet induced by $\Phi$ and apply Whitney's extension theorem, as in the proof of the main theorem). By (b) of Lemma 5.3, the function $\Phi / G_{p}$ also vanishes on $T$ together with all its derivatives, so that a solution $v$ in $C^{\infty}(\bar{D})$ of $\bar{\partial} v=\Phi / G_{p}$ can be found. Now it is enough to define $f_{p}=\phi-v G_{p}$ (the properties of $G_{p}$ imply that $f_{p}$ satisfies all requirements).

2. Now we apply a procedure used by Malgrange to prove Whitney's extension theorem in the $C^{\infty}$-case (see [7]). With the notations of Lemma 5.4, for each $p \in N$, the function $f_{\bar{p}+1}-f_{\bar{p}}$ satisfies the hypotheses of that lemma, and so there 
exists $k_{p} \in A^{\infty}$ vanishing on $E$ together with all its derivatives such that

$$
\left\|f_{\bar{p}+1}-f_{\bar{p}}-k_{p}\right\|_{A^{p}}<1 / 2^{p} \text {. }
$$

Define now $f=f_{\overline{1}}+\sum_{p \geqslant 1} f_{\bar{p}+1}-f_{\bar{p}}-k_{p}$. For fixed $q \in N$, we can write

$$
f=f_{\bar{q}+1}-\sum_{p=1}^{q} k_{p}+\sum_{p>q}\left(f_{\bar{p}+1}-f_{\bar{p}}-k_{p}\right) \text {. }
$$

But, $\left\|f_{\bar{p}+1}-f_{\bar{p}}-k_{p}\right\|_{A^{q}} \leqslant\left\|f_{\bar{p}+1}-f_{\bar{p}}-k_{p}\right\|_{A^{p}}<1 / 2^{p}$ for $p>q$. Hence, the last series is convergent in $A^{q}$ and shows that $f \in A^{q}$. Since this is true for every $q$, $f \in A^{\infty}$. Finally, the expression (26) also shows that $f$ interpolates in $E$ all derivatives of $\phi$. This is so because $f_{\bar{q}+1}$ interpolates the first $\bar{q}$-derivatives and the other terms vanish on $E$ together with their first $\bar{q}$ derivatives.

VI. An example. In this section we give example of an ATW-set which is not a $K$-set, and such that $\log \rho \in$ BMO. It is the following,

$$
E=\{\exp (i / n), n>1\} \cup\{1\} .
$$

The lengths of the complementary intervals of $E$ are $\rho_{n}=1 / n(n+1)$.

1. $E$ is not a $K$-set. For if $I=\left\{e^{i t}: 0 \leqslant t \leqslant 1 / n\right\}$ we have $|I| \sim 1 / n$ and $\sup \{\rho(x), x \in I\} \sim 1 / n(n+1)$.

2. $E$ is an ATW-set. It is easily seen that condition (ATW) is equivalent to

$$
\sum \rho_{k} \log \left(1 / \rho_{k}\right) \leqslant\left(\sum \rho_{k}\right)\left(A \log \left(1 / \Sigma \rho_{k}\right)+B\right)
$$

for every finite family of consecutive $\left(a_{k}, b_{k}\right), \rho_{k}=b_{k}-a_{k}$. In our case this means that we must verify

$$
\sum_{k=m}^{n} \frac{\log k(k+1)}{k(k+1)} \leqslant\left(\sum_{k=m}^{n} \frac{1}{k(k+1)}\right)\left(-A \log \sum_{k=m}^{n} \frac{1}{k(k+1)}+B\right) .
$$

But

$$
\sum_{k=m}^{n} \frac{1}{k(k+1)} \sim \frac{n-m+1}{n(m-1)}
$$

and

$$
\sum_{k=m}^{n} \frac{\log k(k+1)}{k(k+1)} \leqslant \int_{m-1}^{n} \frac{\log x(x+1)}{x(x+1)} d x \leqslant 2\left(\frac{1+\log (m-1)}{m-1}-\frac{1+\log n}{n}\right) .
$$

Then, inequality (27) follows from the inequality

$$
\frac{\log m}{m}-\frac{\log n}{n} \leqslant \frac{n-m}{n m}\left(A \log \frac{n m}{n-m}+B\right) \text {. }
$$

3. $\log \rho \in$ BMO. In fact, $\rho^{\varepsilon}$ is in the Muckenhoupt class $A_{2}$ (see [8]) if $\varepsilon<1 / 2$ :

Fix $0<\varepsilon<1 / 2$. We have to see that

$$
I\left(\rho^{\varepsilon}\right) I\left(\rho^{-\varepsilon}\right)<\text { const. }
$$

We verify this for different classes of intervals.

(a) $I$ has its endpoints in $E$. If $I=\left\{e^{i t}: 1 / n \leqslant t \leqslant 1 / m\right\}$ it is easily seen that

$$
\int_{I} \rho^{\varepsilon} \leqslant \operatorname{const}\left(m^{-1-2 \varepsilon}-n^{-1-2 \varepsilon}\right), \quad \int_{I} \rho^{-\varepsilon} \leqslant \operatorname{const}\left(m^{2 \varepsilon-1}-n^{2 \varepsilon-1}\right),
$$


and so, $\left(\int_{I} \rho^{\varepsilon}\right)\left(\int_{I} \rho^{-\varepsilon}\right) \leqslant \operatorname{const}(n-m)^{2} / n^{2} m^{2} \sim$ const $|I|^{2}$. The case $n=\infty$ is also included here.

(b) Those $I$ for which there exists $a \in E$ such that $\rho(x)=|x-a|$ for $x \in I$. For these $I$ it is easy to see that

$$
\int_{I} \rho^{\varepsilon} \sim\left(\sup _{x \in I} \rho(x)\right)^{\varepsilon}|I|, \quad \int_{I} \rho^{-\varepsilon} \sim\left(\sup _{x \in I} \rho(x)\right)^{-\varepsilon}|I|,
$$

so that (28) follows again.

(c) Those $I$ contained in a component of $T \backslash E$ and containing the middle point of this component. It is not difficult to see that the relations (29) also hold in this case.

(d) Now just one case remains. Those $I=\left\{e^{i t}: a \leqslant t \leqslant b\right\}$ such that $1 / n \leqslant a \leqslant$ $1 / n-1,1 / m \leqslant b \leqslant 1 / m-1$, with $n>m$, and if $n=m+1, a$ is in the left half of $[1 / n, 1 / n-1]$ and $b$ in the right half of $[1 / m, 1 / m-1]$.

We write $I^{\prime}=\left\{e^{i t}: 1 / n \leqslant t \leqslant 1 / m\right\}$. Then, $I \subset I^{\prime}$ and $\left|I^{\prime}\right| \sim\left|I^{\prime}\right|$.

Hence,

$$
\left(\int_{I} \rho^{\varepsilon}\right)\left(\int_{I} \rho^{-\varepsilon}\right) \leqslant\left(\int_{I^{\prime}} \rho^{\varepsilon}\right)\left(\int_{I^{\prime}} \rho^{-\varepsilon}\right) \leqslant \text { const }\left|I^{\prime}\right|^{2} \leqslant \text { const }|I|^{2}
$$

and (28) follows again.

Acknowledgement. I am grateful to Professor A. M. Chollet for posing the problem, as well as for her kind advice and encouragement.

\section{REFERENCES}

1. H. Alexander, B. A. Taylor and D. L. Williams, The interpolating sets for $A^{\infty}$, J. Math. Anal. Appl. 36 (1971), 556-568.

2. A. Baernstein II, Analytic functions of bounded mean oscillation (preprint).

3. L. Carleson, Sets of uniqueness for functions regular in the unit circle, Acta Math. 87 (1952), 325-345.

4. P. L. Duren, Theory of $H^{p}$ spaces, Academic Press, New York, 1970.

5. E. M. Dynkin, Free interpolation sets for Hölder classes, Mat. Sb. 109 (151) (1979), 107-128 = Math. USSR-Sb. 37 (1980), 97-117.

6. F. John and L. Nirenberg, On functions of bounded mean oscillation, Comm. Pure Appl. Math. 14 (1961), 415-426.

7. B. Malgrange, Ideals of differentiable function, Tata Institute, Bombay, 1966.

8. D. Sarason, Function theory on the unit circle, mimeographed notes of lectures, Dept. of Math., Virginia Polytechnic Institute, 1978.

9. E. M. Stein, Singular integrals and differentiability properties of functions, Princeton Univ. Press, Princeton, N. J., 1970.

10. B. A. Taylor and D. L. Williams, Zeros of Lipschitz functions analytic in the unit disc, Michigan Math. J. 18 (1971), 129-139.

11. Ideals in rings of analytic functions with smooth boundary values, Canad. J. Math. 22 (1970), 1266-1283.

12. J. Bruna, Les ensembles $d^{\prime}$ interpolation des $A^{P}(D)$, C. R. Acad. Sci. Paris Ser. A 290 (1980), 25-27.

Department de Teoria de Foncions, Secció de Matemàtiques de la Universitat Autònoma de Barcelona, Bellaterra, Barcelona, Spain 